\title{
A Novel Polyester Fabric Coated with Nanoclay for Discoloration of Reactive Red 4 Dye from Aqueous Solution
}

\author{
AMIRHOSSEIN MONZAVI ${ }^{1}$, MAJID MONTAZER $^{2 *}$ and REZA MOHAMMAD ALI MALEK ${ }^{3}$ \\ ${ }^{1}$ Department of Textile Engineering, Science and Research Branch, \\ Islamic Azad University, Tehran, Iran. \\ ${ }^{2}$ Department of Textile Engineering, Functional Fibrous Structures \& Environmental \\ Enhancement (FFSEE), Amirkabir University of Technology, Tehran, Iran. \\ ${ }^{3}$ Department of Textile Engineering, Center of Excellence in Textile, \\ Amirkabir University of Technology, Tehran, Iran. \\ *Corresponding author E-mail: "tex5mm@aut.ac.ir \\ http://dx.doi.org/10.13005/ojc/330450
}

(Received: November 10, 2016; Accepted: April 12, 2017)

\begin{abstract}
Here, a commercially available absorbent with a high adsorption capacity (Cloisite $® 15 \mathrm{~A}$ ) was coated on a relatively cheap and flexible polyester fabric through simple pad-dry-cure method for removing dye from an aqueous solution. By using this component with good activity against discoloration expected to produce a substrate with a good ability discoloration. FESEM images with EDX patterns and X-ray diffraction spectra revealed the presence of nanoclay on the fabric surface. Discoloration of Reactive Red 4 dye (RR4) was performed by addition of a piece of the coated fabric with stirring in the dye solution. The surface of the coated fabric was colored by the presence of nanoclay acts as adsorbent dye.
\end{abstract}

Keywords: Discoloration, Reactive Red 4 dye, Nanoclay, Polyester fabric.

\section{INTRODUCTION}

Reactive dyes are the most important dye for the dyeing of different textile substrates, in particular cellulosic fibers. ${ }^{1,2}$. The wash fastness properties of dyed fabric with reactive dyes are classified as very good ${ }^{3,4}$. Textile dyes are one of the main pollutants in textile effluents by reducing light penetration and dissolved oxygen inhibition aquatic photosynthesis ${ }^{5}$. Even the inefficient fixation and hydrolysis in water leaves large amounts of non-fixed dye and hydrolyzed as effluents ${ }^{6,7}$. The presence of very small amount of the dye is visible in the effluent. Therefore, discoloration of reactive dyes containing textile wastewater before discharge is necessary. The reactive dyes are stable to heat, light and oxidants 8,10 .

The conventional effluent treatments are ineffective for the removal of dye due to the toxicity 
and degradation resistant of reactive dyes ${ }^{11,12}$. Some physical and chemical techniques have been reported for the removal of reactive dyes as ozonation, activated carbon adsorption, biodegradation, and advanced oxidation processes ${ }^{13,16}$. Recently, they have been used advanced oxidation processes with ozone and the ozone-based method as a technology capable of degradation of organic pollutants ${ }^{17,19}$.

Adsorption techniques have also attracted attention in recent years because of their effectiveness in the removal of polluting effluent. Montmorillonite clay typically is to form a very soft phyllosilicate mineral formed in a microscopic crystal. This is made plate-shaped mineral particles with an average diameter of one micron. It is an important component of weathering volcanic ash, bentonite, with the particles of extremely small thickness $(\sim 1 \mathrm{~nm})^{20,24}$.

Yang et al., used nanoclay, montmorillonite, and some nanoclay modified as sorbents for non-ionic, anionic and cationic dyes. They have demonstrated that with some modifications on nanoclay, an excellent sorbent can be obtained for textile dyes ${ }^{25}$. Liu et al., activated raw clays by acid treatment or calcinations and organic-modified clays with small molecules or polymers to adsorb and remove organic dyes from aqueous solutions ${ }^{26}$.

Woven fabric made of polyester (PET) is cheap and flexible, with high mechanical strength and surface area. The construction of the flexible fabric allows the rapid removal of the dye in the solution. In addition, the open design ensures optimal surface and substrate turnover active easily changed from the fiber diameter ${ }^{27,28}$. In this study, nanoclay coat was applied to the PET fabric by pad-dry-cure technique and the structure was demonstrated by FTIR, FESEM and XRD. The reactive dye adsorption on samples described studying the influence of various parameters, including the contact time and adsorbent dosage of the solution. Moreover, the fabric on both sides can be used for the adsorption.

\section{MATERIALS AND METHODS}

Cibacron Brilliant Red 3B-A (Reactive Red 4) was supplied by Sigma-Aldrich Chemie GmbH (Germany) with empirical formula $\mathrm{C}_{32} \mathrm{H}_{24} \mathrm{CIN}_{8} \mathrm{Na}_{4} \mathrm{O}_{14} \mathrm{~S}_{4}$, molar weight of
$1,000.25 \mathrm{~g} / \mathrm{mol}$ and $\lambda \max =517 \mathrm{~nm}$. The chemical structure of the dye is shown in Fig. 1(A). Modified montmorillonite with a quaternary ammonium salt (Cloisite ${ }^{\circledR}$ 15A) was obtained from Southern Clay Products, Inc (USA). The chemical and physical structure of the nanoclay is shown in Fig.1(B). A $100 \%$ polyester woven fabric weighing $97 \mathrm{~g} \mathrm{D} \mathrm{m2}$ with a yarn count of 150 Den and wrap/weft density of 30/22 yarn/cm was purchased from Hejab Textile Co. (Tehran, Iran).

\section{Preparation and coat nano materials on the fabric}

The aqueous dispersion was prepared by mixing 3-20 (w/v \%) nanoclay and distilled water needed in a bath with a liquor goods ratio of $40: 1$ for 15 minutes at $30-40{ }^{\circ} \mathrm{C}$. Non-ionic detergent was used for the washing of polyester fabric before treatment. The washed fabric was impregnated in the dispersion padded with $90 \%$ wet pick-up dried at $100{ }^{\circ} \mathrm{C}$ for 4 minutes followed by curing at $190{ }^{\circ} \mathrm{C}$ for 7 minutes in a thermal oven (Binder, Germany).

\section{Procedure}

First, $0.25 \mathrm{~g}$ coated fabric was cut and placed in the beaker. $25 \mathrm{~mL}$ dye solution with the concentration of $20 \mathrm{ppm}$ was added to the beaker and exposed to stirring for a few minutes. The absorbance spectra of the solution finally registered. The experimental set-up was based on absorbance reaction apparatus made of glass with dimensions of $3 \times 3 \mathrm{~cm}$. dye solution, including fabric coated with nanomaterials poured from the top of the container. The container was washed after each run of the experiment and a new piece of coated fabric was used later.

\section{Analytical procedures}

The aqueous solution absorbance was monitored with a double beam UV/Vis spectrophotometer (Uvikon 923, Bio-Tek Kontron Instruments). Fourier Transform Infrared (FTIR) spectra were registered using the Thermo Nicolet. The microscopic images were obtained from a Field Emission Scanning Electron Microscope (FESEM; MIRA3 XMU, Tescan). Energy dispersive X-ray spectroscopy (EDS) point analysis and mapping with the Oxford AZTEC EBSD-EDS system was performed. X-ray diffraction analysis from the Seifert XRD 3003 PTS diffractometer system was used, 
with the Rayflex software. Different fabric samples prepared according to Table 1.

Table 1: Fabric preparation for discoloration, including nanaoclay $15 \mathrm{~A}$

\begin{tabular}{lccccc}
\hline Fabric No. & 1 & 2 & 3 & 4 & 5 \\
$15 \mathrm{~A}(\mathrm{w} / \mathrm{v} \%)$ & 3.00 & 6.45 & 11.50 & 16.55 & 20.00 \\
\hline
\end{tabular}

Nanoclays modified with a quaternary ammonium salt is used as a strong adsorbing dye. However, if the layers of nanoclay exfoliation, the adsorption capacity increases dramatically. With the opening of the clay layers by stirring in the treatment bath (Fig.2), the dye molecules adsorbed to the layers. Witch stirring nanoclays are well dispersed in an aqueous medium. Through the exfoliation of the nanoclay layers, the ammonium salts are situated between the plates.

\section{RESULTS AND DISCUSSION}

After impregnation of the fabric in the dispersion solution, the fabric was padded with the indicated wet pickup, dried and cured to fix the nanomaterials on the substrate. The distribution of the nanoparticles on the fabric substrate is shown in Fig.2.

\section{FTIR Analysis}

The FTIR spectra of the polyester fabric before and after coat with nanoclay are illustrated in Fig.3. The saturated esters related peaks were found at $1729 \mathrm{~cm}^{-1}$ due to carbonyl $(-\mathrm{CP}=\mathrm{O})$ stretching. The bands around 1330-1240 and 1160-1080 $\mathrm{cm}^{-1}$ is due to vibration of the ester group ${ }^{29}$. Also the $-\mathrm{C}-\mathrm{H}$ bonds linked with the benzene ring in the polymer structure show shades peaks around $1600-1400 \mathrm{~cm}^{-1}$ with beyond the plane of the benzene ring at 1018, 875 and $730 \mathrm{~cm}^{-1} 30$. The characteristic bands of nanoclay can be observed prior to $1100 \mathrm{~cm}^{-1}$. A peak at $1020 \mathrm{~cm}^{-1}$ belongs to the stretching vibration of $\mathrm{Si}-\mathrm{O}$ bonds of the tetrahedral silica layers. The peaks at 719,619 , 519 and $451 \mathrm{~cm}^{-1}$ correspond to Si-O and Al-O bending vibration of the octahedral and tetrahedral layers of silica-alumina. The bands have appeared at 2845 and $2920 \mathrm{~cm}^{-1}$ are specific to stretching vibration of $-\mathrm{CH}_{2}$ and $-\mathrm{CH}_{3}$ of alkyl ammonium cation, respectively ${ }^{31}$. The $-\mathrm{CH}$ bending vibration of $-\mathrm{CH}_{3}$ is also observed at $1465 \mathrm{~cm}^{-1}$. The stretching vibration of $-\mathrm{OH}$ groups are characterized by a band at $3622 \mathrm{~cm}^{-1} 32$.

\section{FESEM images}

FESEM images of various polyester fabrics including untreated and treated with nanoclay are presented in Fig.3. This is used to confirm the load nanoparticles on the surface of the substrate for the additional dye removal. Fig.3(a) showed the surface of the polyester fiber (control sample) with a clean surface and not loaded nanoparticles. According to Fig.3(b) the nanoclay coated sample compared to the uncoated sample which nanoclay loaded on the fabric surface. To estimate the average thickness of nanoclay layer distributed on the fabric surface, SEM images were taken at $75.0 \mathrm{KX}$. It is revealed that the nanoclay plates with a thickness of about 10 $\mathrm{nm}$ are uniformly distributed on the fabric surface. SEM image of 3(b) has confirmed that the surface characteristics of the fabrics modified after loading and one layer of nanoclay extensively covered,

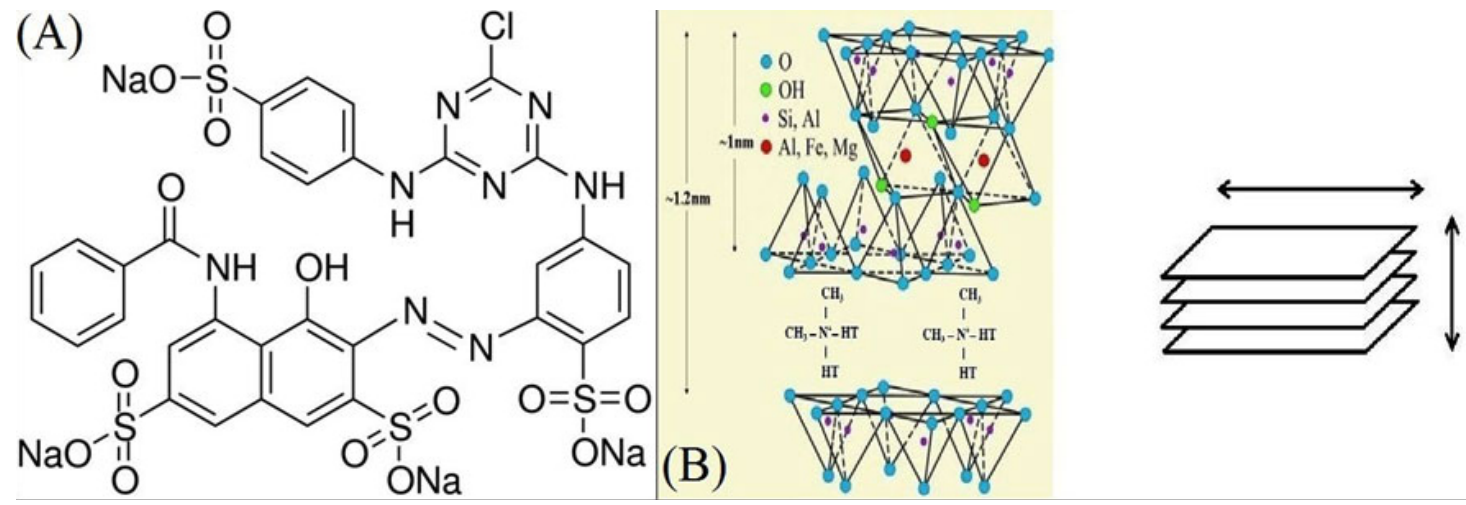

Fig. 1: The chemical structure of (A) CI Reactive Red 4 and (B) Cloisite ${ }^{\circledR}$ 15A. 
which implies an even loading of nanomaterials. The average thickness of nanoclay layer observed about $10-11 \mathrm{~nm}$.

\section{EDS pattern}

The presence of nanomaterials on the fabrics structure was investigated by energy dispersive $\mathrm{X}$-ray spectroscopy. EDS spectrum of polyester fabric sample treated with the nanoclay was examined to understand the basic specification of the fabric (Fig.3). Photoelectron peaks were observed at about $200 \mathrm{eV}$, it shows the presence of $\mathrm{Si}, \mathrm{Al}$ and $\mathrm{Mg}$, which is another proof of nanoclay particles on the fabric surface and the presence of $\mathrm{C}$ and $O$ is due to the polyester chains. The presence of nanoclay on the surface of the treated fabric has been confirmed that the sign of $\mathrm{Si}, \mathrm{Al}$ and $\mathrm{Mg}$ peaks indicated on rough fabric. Au peaks are also included in the spectrum as the processed sample was coated with gold before EDS study.

\section{X-ray Diffraction}

The XRD patterns of different samples, including control and treated fabric illustrated in Fig.3. The polyester characteristic peaks in the spectra are identified with the star at Bragg angles of 17.62, 23.11 and $25.57^{\circ}{ }^{33}$. Two new peaks at 5.04 and $6.69^{\circ}$ denoted the presence of nanoclay on the fabric surface $^{34}$. It can be seen that $d(001)$ than the raw clay presented to $7.64^{\circ}$, which corresponds to the layer spacing of $1.15 \mathrm{~nm}$. The method to be of clay on the fabric surface is determined according to the evolution and the intensity peak. The appearance of new peaks at 5.49 and $5.72^{\circ}$ in the treated sample with nanoclay $\mathrm{d}=1.6$ and $1.7 \mathrm{~nm}$ represented the increasing distance between the corresponding clay layers. Therefore, the presence of the quaternary ammonium salt has led to the intercalation between the clay sheets. Other reflections have appeared in the XRD pattern attributed to disorientation hydrogenated tallow alkyl between the clay sheets. Despite these diffraction peaks can potentially produce more nanoclay content on the fabric, which led the aggregations, however, the degree of aggregation were quite low due to the low intensity of diffraction peaks at $4.64^{\circ}$. The other peaks decreased slightly and shifted due to delaminating and confusion of clay sheets ${ }^{35}$.

The large surface area to mass ratio of nanomaterials significantly improves the adsorption capacity as the absorbent. In addition to high specific surfaces, nanoparticles have exclusive adsorption due to different distributions of reactive surface sites and disturbed surfaces. Adsorption of organics to the nanoclay media has been extremely rapid.

The most important absorbance peak in the visible spectrum of Cibacron Brilliant Red 3B-A can be specified at $517 \mathrm{~nm}$ and can be used to measure the discoloration of the dye solution.The solution adsorption values for different conditions of stirring time are shown in Fig. 4. The dye concentration in the solution indicates that the adsorption equilibrium of the dye to the treated fabric is greater than that of the untreated one. On this basis, nanoclay increases adsorption of the dye on the fabric and acts as the most important criteria in the discoloration.

The changes in the UV-visible spectrum of reactive dye solutions were monitored to investigate the mechanism responsible for the discoloration. The results are shown in Fig.4. Adsorption of reactive dyes of clay nanosheets is due to chemical

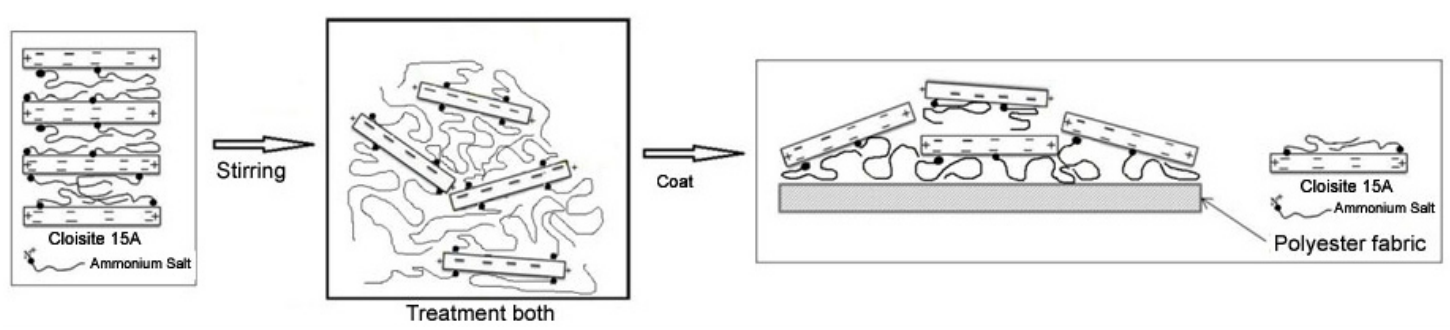

Fig.2: Schematic of interaction between nanoclays, ammonium salts and polyester fabric. After impregnation of the fabric in the dispersion solution, the fabric was padded with the indicated wet pickup, dried and cured to fix the nanomaterials on the substrate. The distribution of the nanoparticles on the fabric substrate is shown in Fig.2. 

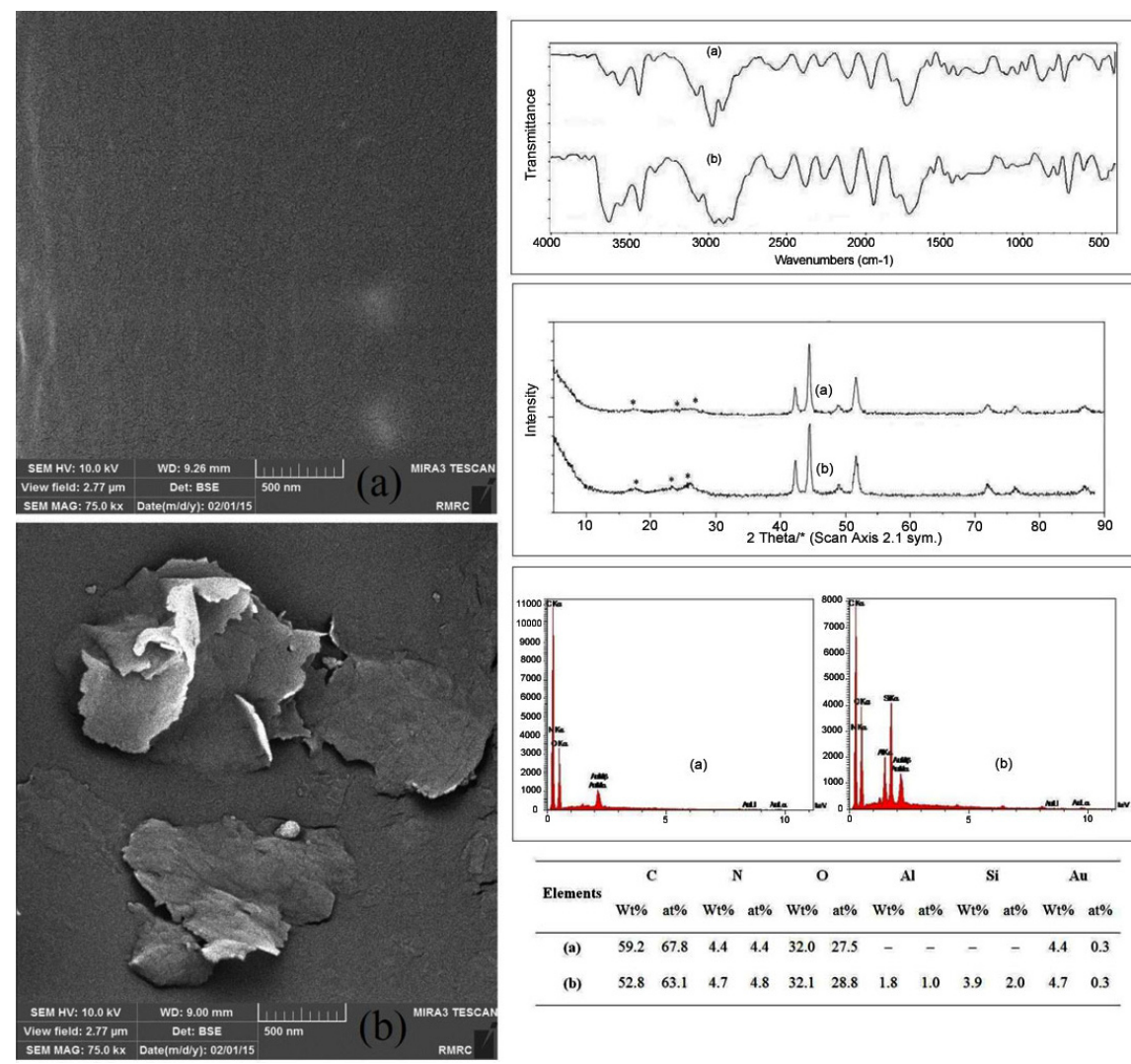

Fig. 3: FESEM images, FTIR spectra, XRD and EDS patterns of various polyester fabrics (a) untreated and (b) coated with nanoclay.

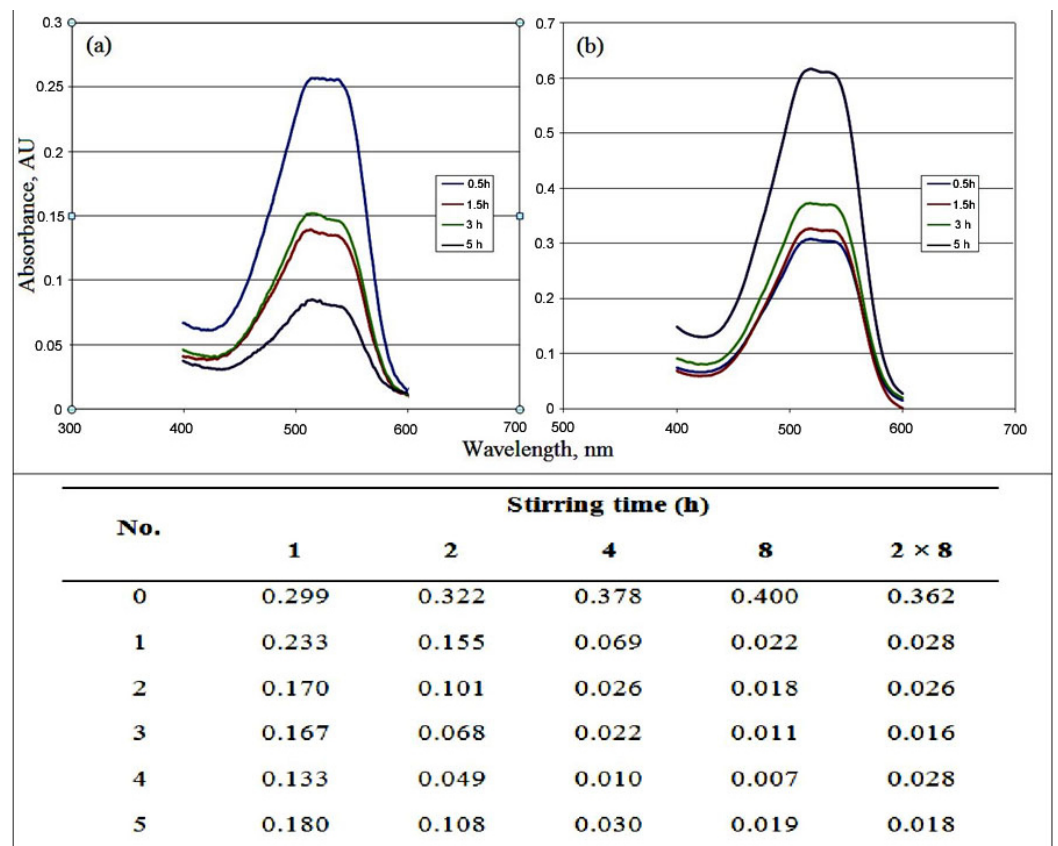

Fig.4: Absorbance spectra of dye solutions [Cl Reactive Red 4 (20 ppm)] discolored using polyester fabric treated with (a) nanoclay and the control sample (b) under stirring. 
interaction between the dyes and distribution of the various functional units in the interlayer spacing of two dimensional layered nanoclays.

The adsorption of dye solutions depends on many factors including the type, $\mathrm{pH}$ and the concentration of the dye. At high concentrations of reactive dyes used in the present study (20 ppm), the adsorption capacity may be low.

The results in Fig.4 also show that the adsorption of dye increases with an increase of the stirring time. The appearance of red color on polyester fabric coated with the dispersion of nanoclay is a visible proof of the greater ability of dye sorption. The chemisorptions of the anionic reactive dyes on polyester treated with nanoclay mainly due to the electrostatic attraction between the nanoclay ammonium salt groups and fully deprotonated sulfonic groups of the dye solutions ${ }^{36}$. During the periods of discoloration, the peak of the maximum wavelength was stable. Thus, by coating the polyester substrate with nanoclay sheets, adsorption initially played a dominant role for dye removal. As the discoloration was completed at the end of the shaking, the absorbance peak for all the procedures is roughly disappeared, and a strong red dye solution became colorless.

\section{CONCLUSION}

The textile materials have been successfully introduced as an excellent substrate for the immobilization of nanomaterials on the surface to remove the dye from dyeing effluents. Adsorption of Reactive Red 4 from the aqueous solution was carried out in the presence of polyester fabric with immobilized nanoclay layers by stirring. Also the stability of nanomaterials on the fabric surface was good. The process of decolorization of the dye solution is repeated with the same substrate several times with reasonable efficiency discoloration. In this study, the objective of the dye desorption further confirms that adsorption is primarily responsible for the initial discoloration.

\section{REFERENCES}

1. Dolby, P.J. Textile Chemist \& Colorist, 1977, 9, 32-36

2. Khatri, A.; Peerzada, M.H.; Mohsin, M.;White, M. J. Clean. Prod. 2015, 87, 50-57

3. Zhang, S.; Ma, W.; Ju, B.; Dang, N.; Zhang, M.;Wu, S.; Yang, J. Color. Technol. 2005, 121, 183-186

4. Rizk, H.F.; Ibrahim, S.A.; El-Borai, M.A. Dyes Pigments, 2015, 112, 86-92

5. Correia, V.M.; Stephenson, T.; Judd, S.J. Environ. Technol. 1994, 15, 917-929

6. Robinson, T.; Mc Mullan, G.; Marchant, R.; Nigam, P.Bioresource Technol. 2001, 77, 247-255

7. Kordouli, E.; Bourikas, K.; Lycourghiotis, A.; Kordulis, C. Catal. Today, 2015, 252, 128-135

8. Renfrew, A.H.M.; Taylor, J.A. Color. Technol. 1990, 20, 1-9

9. Fujioka, S.; Abeta, S. Dyes Pigments, 1982, 3, 281-294

10. Lucic, M.; Milosavljevic, N.; Radetic, M.; Saponjic, Z.; Radoicic, M.; Krusic, M.K. Sep.
Purif. Technol. 2014, 122, 206-216

11. Crini, G. Bioresource Technol. 2006, 97, 1061-1085

12. Chong, M.N.; Cho, Y.J.; Poh, P.E.; Jin, B. J. Clean. Prod. 2015, 89, 196-202

13. Ezechi, E.H.; Kutty, S.R.M.; Malakahmad, A.; Isa, M.H. Process Saf. Environ. 2015, 98, 16-32

14. Abidi, N.;Errais, E.;Duplay, J.;Berez, A.;Jrad, A.; Schafer, G.; Ghazi, M.;Semhi, K.;TrabelsiAyadi, M. J. Clean. Prod. 2015, 86, 432-440

15. Soboleva, N.M.;Nosovich, A.A.;Goncharuk, V.V. J. Water Chem. Technol. 2007, 29, 72-89

16. Franz, S.;Perego, D.;Marchese, O.;Bestetti, M. J. Water Chem. Technol. 2015, 37, 108-115

17. Azbar, N.;Yonar, T.;Kestioglu, K. Chemosphere, 2004, 55, 35-43

18. Radi, M.A.;Nasirizadeh, N.;RohaniMoghadam, M.;Dehghani, M. Ultrason. Sonochem. 2015, 27, 609-615

19. Khouni, I.;Marrot, B.; Moulin, P.; Amar, R.B. Desalination, 2011, 268, 27-37 
20. Elemen, S.;Kumbasar, E.P.A.;Yapar, S. Dyes Pigments, 2012, 95, 102-111

21. Zhou, C.H.; Keeling, J. Appl. Clay Sci. 2013, 74, 3-9

22. Duman, O.;Tunc, S.;Polat, T.G. Appl. Clay Sci. 2015, 109-110, 22-32

23. Ghemati, D.j.;Aliouche, D.j. J. Water Chem. Technol. 2014, 36, 265-272

24. Nadafi, K.;Vosoughi, M.;Asadi, A.;Omidvar Borna, M.;Shirmardi, M. J. Water Chem. Technol. 2014, 36, 125-133

25. Yang, Y.; Han, S.; Fan, Q.; Ugbolue, S.C. Text. Res. J. 2005, 75, 622-627

26. Liu, P.; Zhang, L. Sep. Purif. Technol. 2007, 58, 32-39

27. Radetic, M. J. Photoch. Photobio. C.2013, 16, 62-76

28. Farouk, A.; Sharaf, S.; El-Hady, M.M.A. Int. J. Biol. Macromol.2013, 61, 230-237
29. Cole, K.C.; Daly, H.B.;Sanschagrin, B.; Nguyen, K.T.;Ajji, A. Polymer, 1999, 40, 3505-3513

30. Mejia, M.I.; Marin, J.M.; Restrepo, G.; Rios, L.A.;Pulgarin, C.; Kiwi, J. Appl. Catal. B-Environ. 2010, 94, 166-172

31. Dalir, H.; Farahani, R.D.;Nhim, V.; Samson, B.; Levesque, M.;Therriault, D. Langmuir, 2012, 28, 791-803

32. Kozak, M.;Domka, L. J. Phys. Chem. Solids, 2004, 65, 441-445

33. Chang, J.H.; Kim, S.J.;Joo, Y.L.;Im, S. Polymer, 2004, 45, 919-926

34. Phang, I.Y.;Pramoda, K.P.; Liu, T.; He, C. Polym. Int. 2004, 53, 1282-1289

35. Asadi, M.;Montazer, M. J. Inorg. Organomet. P. 2013, 23, 1358-1367

36. Wangpradit, R.;Chitprasert, P. Int. Biodeter. Biodegr. 2014, 93, 168-176 